\title{
Hepatoma in a young adult: to biopsy or not to biopsy?
}

\section{CASE REPORT}

A 23 year old woman presented with a four month history of epigastric pain, abdominal distention, nausea, and occasional bloodstained vomiting. She had lost $7 \mathrm{~kg}$ in weight. She had a complex cardiac history requiring cardiac surgery for tricuspid atresia at the age of three years and again when she was 20 years.

When she was 16 years, she was diagnosed as being hepatitis $\mathrm{C}$ positive. Her medications included warfarin.

Significant clinical findings included the presence of jaundice without other stigmata of chronic liver disease. She had a systolic murmur with normal metallic sounds from the prosthetic mitral valve. A pacemaker was in situ. She had right upper quadrant tenderness with a palpable liver edge.

Liver function tests revealed a bilirubin level of $79 \mu \mathrm{mol} / \mathrm{l}$ (normal range $0-17)$, alkaline phosphatase of $834 \mathrm{u} / \mathrm{l}$ (30-130), and alanine transaminase of $193 \mathrm{u} / \mathrm{l}(0-31)$. Haemoglobin concentration was $8.7 \mathrm{~g} / \mathrm{dl}$ (12-16). International normalised ratio was 10 . Viral serology was positive for hepatitis $C$ only. Alpha fetoprotein (AFP) measured 11000 units/l (normal range 0-7). Beta human chorionic gonadotrophin ( $\beta$-HCG) levels were not elevated. Other laboratory tests were within normal limits.

Upper gastrointestinal endoscopy showed diffuse haemorrhagic gastritis and large oesophageal varices. Ultrasound showed an irregular heterogenous liver with the possibility of a more focal liver lesion, portal vein thrombosis, ascites, and splenomegaly. Therefore, the patient proceeded to computed tomography. A non-calcified lesion was seen in segment 5 (fig 1A) which showed contrast enhancement most marked on the portal venous phase of imaging. Enhancing thrombus in the portal vein could be seen immediately adjacent to this (fig 1B). A radiological diagnosis of hepatocellular carcinoma (HCC) and portal vein tumour thrombus, on a background of chronic liver disease, was made. Other diagnoses were considered unlikely. However, in view of the patient's age, and the concern that hepatitis $C$ acquired in childhood does not usually lead to early complications, ${ }^{1}$ ultrasound guided biopsy was performed.

Histology revealed a tumour composed of acinar/tubular elements within a desmoplastic stroma. Some of the cells contained mucin and the overall appearances were not typical of HCC. Cells stained with antibodies against epithelial membrane antigen and cytokeratins 7 and 19, with focal staining with antibodies to carcinoembryonic antigen. There was no staining for cytokeratin 20, CD30, CD 1 17, AFP, or $\beta$-HCG. Overall the features were difficult to interpret but favoured a germ cell tumour (GCT) with yolk sac elements rather than a HCC.

Surgical opinion confirmed that the tumour was irresectable on grounds of the portal vein invasion and so the patient was treated with a MBOP chemotherapy regimen (methotrexate, bleomycin, vincristine, and cisplatin).
Her AFP levels decreased by $75 \%$ but she became neutropenic, septic, developed renal failure, and died 10 days after the start of treatment.

\section{DISCUSSION}

HCC is usually diagnosed by a combination of clinical background, imaging findings, and an elevated AFP. These tumours are not routinely biopsied although controversy surrounds the topic. Our case illustrates an example where these features were present but where a biopsy changed the diagnosis, prognosis, and treatment.

HCC is a rare occurrence in the first four decades of life except in areas where hepatitis B is hyperendemic. It is associated with underlying cirrhosis in $70-90 \%$ of cases, with hepatitis $\mathrm{C}$ cirrhosis more likely to lead to HCC than hepatitis B or alcohol related disease. ${ }^{2}$ While the natural history of hepatitis $C$ acquired in childhood is progression to a similar histology as adults, the changes are milder and severe hepatitis or cirrhosis are infrequent complications in adoles- cence. ${ }^{1}$

There are no imaging features unique to HCC. At computed tomography, tumour thrombus can be differentiated from bland thrombus by expansion of the portal vein and enhancement of the thrombus. Angiography and radionuclide imaging are unlikely to further advance the diagnosis. Magnetic resonance imaging (contraindicated in this patient) with mangafodipir trisodium (Teslascan, Nycomed), helps differentiate a tumour containing hepatocytes
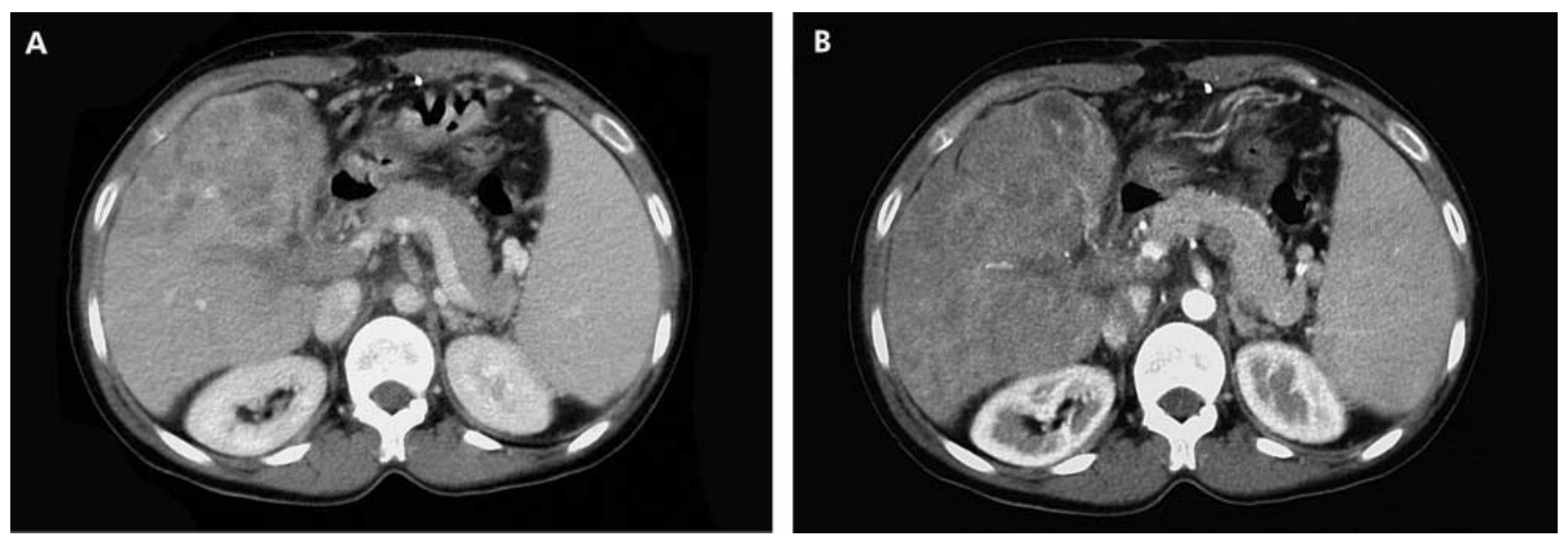

Figure 1 (A) Axial computed tomography scan at 25 seconds post contrast injection, showing a heterogenous poorly enhancing mass in segment 5 of the liver. The portal vein is distended. (B) Axial computed tomography scan at the same level as (A), at 65 seconds post contrast injection. The level of the portal vein obstruction is clearly delineated. There is enhancement of the distended portal vein in keeping with tumour thrombus. The tumour itself is more clearly seen. 
from those which do not, but as hepatic GCT may contain hepatocytes, in theory, differentiation from HCC may still have been impossible.

GCT may be undifferentiated (dysgerminoma, seminoma), or differentiate along embryonic (teratomas, mature, or immature) or extraembryonic (yolk sac or endodermal sinus tumours, choriocarcinomas) lines. ${ }^{3}$ Extragonadal GCT originate from the primitive germ cell line and are usually midline tumours. ${ }^{3}{ }^{4}$ They are classified by the International Germ Cell Cancer Collaborative Group into prognostic groups, based on histology described according to the WHO. ${ }^{5}$ They typically occur in children and young adults. ${ }^{4}$ Extragonadal GCT may be benign or malignant, and may show a variety of tissue types. Histologically, yolk sac tumours have a higher malignant potential than other tumour types. ${ }^{4}$

Elevations in serum $\beta$-HCG are seen with choriocarcinomas while elevated AFP levels occur with yolk sac tumours. ${ }^{4}$

GCT of the liver are extremely rare in adults. ${ }^{6}$ Only four adult pure yolk sac tumours of the liver have been previously reported. ${ }^{7}$ The imaging features of hepatic GCT have not been well described. The production of AFP by hepatic GCT has been reported to range from 500 to $28470 \mathrm{ng} / \mathrm{ml}$ (international units/ litre $\approx 1: 1$ with $\mathrm{ng} / \mathrm{ml}) .^{78}$

Treatment is initially with cisplatin based chemotherapy. Since institution of chemotherapy, five year overall survivals of $60 \%$ have been reported. ${ }^{5}$

In conclusion, radiologists and gastroenterologists, particularly in specialist centres, should be aware that in a very small subset of patients-that is, the young patient with hepatitis C-the classical clinical and imaging findings of cirrhosis and HCC may have another interpretation with a very different prognosis.

C Brenner, Z Amin,

Department of Radiology, Imperial College Faculty of Medicine, Chelsea and Westminster Hospital, London, UK

A C Wotherspoon,

Department of Histopathology, Royal Marsden Hospital, London, UK

P Vlavianos, J Andreyev,

Department of Medicine and Therapeutics, Imperial College Faculty of Medicine, Chelsea and Westminster Hospital, London, UK
Correspondence to: Dr J Andreyev, Department of Medicine and Therapeutics, Chelsea and Westminster Hospital, 369 Fulham Rd, London SW109NH, UK; j.andreyev@ic.ac.uk.

\section{REFERENCES}

1 Hardikar W. Advances in paediatric gastroenterology and hepatology: hepatitis $C$ in childhood. J Gastroenterol Hepatol 2002; 17:476-81.

2 El-Serag HB. Epidemiology of hepatocellular carcinoma. Clin Liver Dis 2001;5:87-107.

3 Harms D, Janig U. Germ cell tumours of childhood. Virchows Arch (Pathol Anat) 1986:409:223-39.

4 Talerman A. Germ cell tumours. Ann Pathol 1985;5:145-57.

5 Dueland S, Stenwig AE, Heilo A, et al. Treatment and outcome of patients with extragonadal germ cell tumours - the Norwegian Radium Hospital's experience 1979-1994. Br J Cancer 1998:77:329-35.

6 Theegarten D, Reinacher A, Graeven U, et al. Mixed malignant germ cell tumor of the liver. Virchows Arch 1998;433:93-6.

7 Narita T, Moriyama Y, Ito Y. Endodermal sinus (yolk sac) tumour of the liver. A case report and review of the literature. I Pathol 1988;155:41-7.

8 Villaschi S, Balistreri P. Endodermal sinus tumour of the liver. Histopathology $1991 ; 18: 86-8$. 